PHYSICAL REVIEW D 93, 089904(E) (2016)

\title{
Erratum: Global solutions of functional fixed point equations via pseudospectral methods \\ [Phys. Rev. D 91, 105011 (2015)]
}

\section{J. Borchardt and B. Knorr}

(Received 18 March 2016; published 8 April 2016)

DOI: 10.1103/PhysRevD.93.089904

We noticed a typo in our code in one of the equations for the critical exponents for the $\mathrm{O}(1)$ model (Sec. V). The actual values of the critical exponents are as follows:

(i) Wilson-Fisher fixed point (Fig. 3): The values are in agreement with recent studies [1].

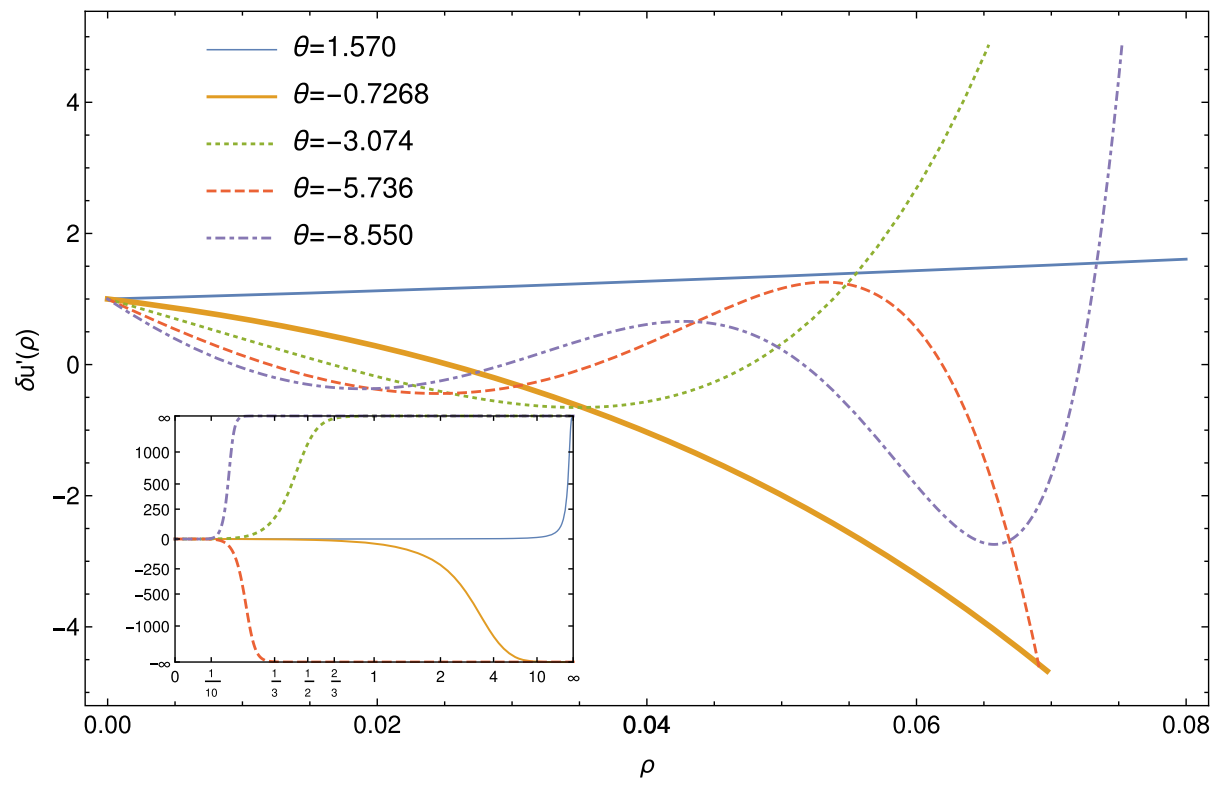

FIG. 3. Eigenperturbations of the Wilson-Fisher fixed point, normalized to one at $\rho=0$.

(ii) Multicritical fixed points in $d=2.4$ :

TABLE I. Anomalous dimensions and highest critical exponents of all scaling solutions in $d=2.4$.

\begin{tabular}{lcr}
\hline \hline$\eta$ & Relev. Exp. & Irrelev. Exp. \\
\hline 0.1390 & WF-FP & -1.0977 \\
& 1.1883 & -3.1108 \\
& & -5.6147 \\
0.01598 & Multicritical FP ${ }_{i=3}$ & -0.5467 \\
& 1.9636 & -2.0694 \\
& 0.8507 & -3.8156 \\
0.001753 & Multicritical FP & \\
& 1.9968 & -0.3035 \\
& 1.4607 & -1.3957 \\
$8.2715 \times 10^{-5}$ & 0.6632 & -0.1555 \\
& Multicritical FP & -0.9284 \\
\end{tabular}




\section{ACKNOWLEDGMENTS}

We would like to thank I. Boettcher for making us aware of this error in the equations.

[1] I. Boettcher, Phys. Rev. E 91, 062112 (2015). 